\title{
Tractable Parameterizations for the Minimum Linear Arrangement Problem
}

\author{
Michael R. Fellows ${ }^{1}$, Danny Hermelin ${ }^{2}$, \\ Frances Rosamond ${ }^{1}$, and Hadas Shachnai ${ }^{3}$ \\ 1 School of Engineering and IT, Charles Darwin University, Darwin, Australia. \\ Email: \{michael.fellows, frances.rosamond\}@cdu.edu.au \\ 2 Department of Industrial Engineering and Management, Ben-Gurion University, \\ Beer-Sheva, Israel. Email: hermelin@bgu.ac.il \\ 3 Computer Science Department, Technion, Haifa 32000, Israel. \\ Email: hadas@cs.technion.ac.il
}

\begin{abstract}
The Minimum Linear Arrangement (MLA) problem asks to embed a given graph on the integer line so that the sum of the edge lengths of the embedded graph is minimized. Most layout problems are either intractable, or not known to be tractable, parameterized by the treewidth of the input graphs. We investigate MLA with respect to three parameters that provide more structure than treewidth. In particular, we give a factor $(1+\varepsilon)$-approximation algorithm for MLA parameterized by $(\varepsilon, k)$, where $k$ is the vertex cover number of the input graph. By a similar approach, we describe two FPT algorithms that exactly solve MLA parameterized by, respectively, the max-leaf and edge-clique-cover numbers of the input graph.
\end{abstract}

\section{Introduction}

Given a graph $G=(V, E)$, a linear arrangement is a linear ordering on the set of vertices $V$ of $G$ which is specified by a permutation $\pi: V \rightarrow\{1,2, \ldots,|V|\}$. The cost of the arrangement is defined by $\operatorname{cost}(\pi)=\sum_{(u, v) \in E}|\pi(u)-\pi(v)|$; that is, the cost of the ordering is the sum total of the edge lengths under the ordering. In typical applications one is interested in linear arrangements of low cost. The Minimum Linear Arrangement (MLA) problem is the problem of finding a linear arrangement of minimum cost, and the standard parameterization of the problem is to determine if an input graph $G$ has a layout of cost at most $k$ (the parameter).

MLA is one of the most important and well studied graph ordering problems. It is in some sense closely related to the BANDWIDTH problem, which seeks to minimize the maximum edge length of an ordering of the vertices. It has various applications, most of which stem from the domain of VLSI circuit design. As it was known to be NP-complete already from the mid 70's [12], most of the early work on this problem focused on designing heuristics and approximation algorithms. For a graph with $n$ vertices, the best known approximation ratio for MLA is $O(\sqrt{\log n} \log \log n)$, due to Feige and Lee [5], and Charikar, Hajiaghayi, Karloff 
and Rao [4]. Earlier notable work includes the paper by Rao and Richa [18] who presented an $O(\log n)$-approximation algorithm for the problem, along with another algorithm for planar graphs that achieves a ratio of $O(\log \log n)$. Recently, Ambühl, Mastrolilli, and Svensson [1] showed that MLA does not admit a PTAS unless NP-complete problems can be solved in randomized subexponential time. We refer the reader to [18] for a further account of earlier work on MLA.

In terms of parameterized complexity, the standard parameterization of MLA is trivially fixed-parameter tractable (FPT). Gutin et al. presented in [14] an FPT algorithm which, given a graph on $n$ vertices and $m$ edges, and a parameter $k$, outputs a linear arrangement of cost at most $m+k$, if one exists. Fernau in [11] described efficient bounded search tree FPT algorithms for MLA under its standard parameterization. Bodlaender et al. [3] investigated exact exponential-time algorithms for several ordering problems, including MLA.

Most parameterized problems are FPT parameterized by the treewidth of the input graph. However, graph layout and width problems are a notable exception (but see also [6] for further examples of parameterized problems that are $W$ [1]hard when parameterized by the input treewidth, or even the input vertex cover number). Parameterized by the treewidth of the input graph, BANDwidTH is known to be hard for $W[t]$ for all $t$ (this follows from the results in [2]). Whether something similar holds for MLA is unknown. This general situation motivates studying the complexity of these problems, parameterized by structural parameters even stronger than treewidth, a program that is sometimes called parameter ecology. See [8] for a recent survey of this area.

In this paper, we consider the complexity of MLA parameterized by three structural parameters that have a certain commonality: all three, when bounded, force the input graph $G$ to have a structure that essentially consists of an elaboration of some small (parametrically bounded) "seed" graph $H$, that gives us sufficient information about the entire graph $G$ to be able to derive efficient algorithms. The three graph structural parameters we consider are:

(i) The vertex cover number of $G$, denoted $v c(G)$, which is the size of the smallest set of vertices intersecting every edge in $G$.

(ii) The maximum leaf number of $G$, denoted $m \ell(G)$, which is the maximum number of leaves in any spanning tree of $G$.

(iii) The edge clique cover number of $G$, denoted ecc $(G)$, which is defined to be the minimum number of cliques required to cover all the edges of $G$.

The question of whether MLA is FPT by the vertex cover number of the input graph has been prominently raised in [10], where it is shown that a number of graph layout and width problems such as BANDwidth, Cutwidth and DisTORTION are FPT by this parameter, and this question has been a noted open problem in parameterized algorithmics. Here we offer a partial positive answer by taking an approach that combines parameterization and approximation. One of our main results shows that MLA can be approximated to within a factor of $(1+\varepsilon)$ of optimal, in FPT time for the aggregate parameter $(1 / \varepsilon, k)$, where $k$ is the vertex cover number of the input graph. (Whether the MLA problem can be exactly solved in FPT time for the parameter $k$ alone still remains open.) In [9] 
it is shown that BANDWIDTH is FPT, parameterized by the max leaf number of the input graph. Here we obtain a matching result for MLA; it can be exactly solved in FPT time for this parameter. While the techniques used in both results look similar from a bird's eye, there are several major differences hiding in the details. Finally, our last result shows that the edge clique cover number can potentially be a useful parameterization for other graph layout problems.

The paper is organized as follows. In Section 2 we give a $(1+\varepsilon)$-approximation for MLA parameterized by $v c(G)$. In Sections 3 and 4 we present FPT algorithms for MLA using the parameters $m \ell(G)$ and $\operatorname{ecc}(G)$, respectively. We conclude in Section 5 with some open problems. Due to space constraints some of the proofs are omitted. The detailed results appear in [7].

\section{MLA parameterized by Vertex-Cover Number}

In this section we present an algorithm which yields a $(1+\varepsilon)$-approximation for MLA in FPT-time with respect to $k=v c(G)$ and $1 / \varepsilon$, where $G$ is the input graph and $\varepsilon>0$. Our algorithm proceeds as follows.

W.l.o.g., we assume that $G$ has no isolated vertices, and let $m=|E|$. The first step of our algorithm is to compute a vertex cover $V^{\prime} \subseteq V$ of $G$ of size $k$; let $I=V \backslash V^{\prime}$. Note that each vertex in $I$ has neighbors only in $V^{\prime}$. We define the type of node $u \in I$ to be its set of neighbors, $N(u)$. Clearly, there are $T \leq 2^{k}$ different types of vertices in $I$. We let $n_{t}$ denote the number of vertices of type $t$, $1 \leq t \leq T$. The main idea of our algorithm is as follows. We put together vertices of the same type into groups of an appropriately chosen size, and then compute an optimal linear arrangement for the graph obtained by merging each group into a single mega-vertex. The analysis of our algorithm relies on an interesting homogeneity lemma, which relates to the behavior of vertices of identical type inside "gaps" formed by the vertices of $V^{\prime}$ in an optimal arrangement for $G$. A detailed description of the algorithm is given below (see Algorithm 1).

\subsection{Analysis}

We now prove that the algorithm yields a solution that is within factor $(1+\varepsilon)$ from the optimal.

Theorem 1. Algorithm 1 computes a $(1+\varepsilon)$-approximate linear arrangement for $G$ in FPT time with respect to $k$ and $1 / \varepsilon$.

We use in the proof of the theorem a few lemmas. Given a layout $\pi$ for $G$, let $u_{1}, \ldots, u_{k}$ denote the vertices in $V^{\prime}$ as ordered in $\pi$. We say that a vertex $v \in I$ is in gap $i, 1 \leq i \leq k-1$, if $\pi\left(u_{i}\right)<\pi(v)<\pi\left(u_{i+1}\right)$. Similarly, $v$ is in gap 0 if $\pi(v)<\pi\left(u_{1}\right)$, and in gap $k$ if $\pi\left(u_{k}\right)<\pi(v)$. The following lemma shows that the vertices of $V^{\prime} \backslash V$ appear homogenously according to their type in some optimal linear arrangement of $G$.

Lemma 1 (Homogeneity for $v c(G)$ ). There exists an optimal solution in which the vertices of each type appear in gap $i$ consecutively, for all $1 \leq i \leq k-1$. 


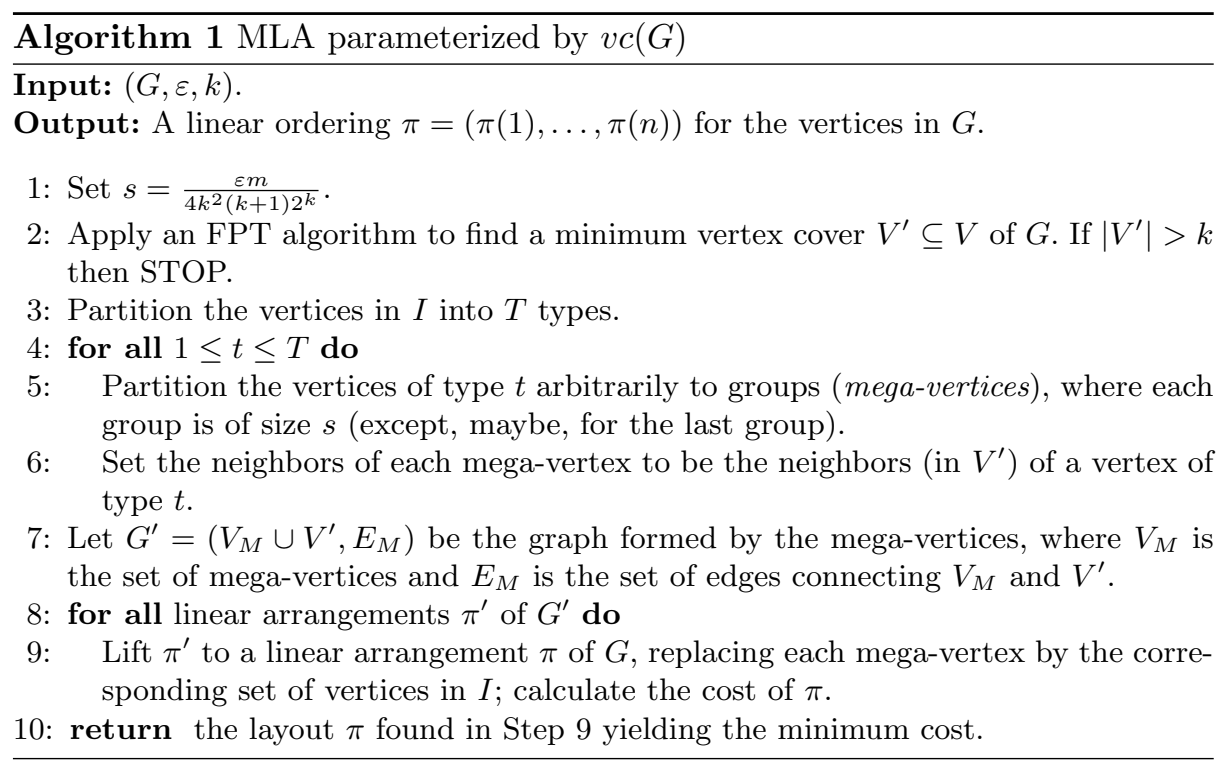

Proof. Let $\pi: V \rightarrow\{1, \ldots, n\}$ denote an optimal linear arrangement of $G$, and let $\delta(v)$ denote the force of $v$ (with respect to $\pi$ ) defined by

$$
\delta(v)=|\{u \in N(v) \mid \pi(u)>\pi(v)\}|-|\{u \in N(v) \mid \pi(u)<\pi(v)\}| .
$$

We note that for any gap $i, 0 \leq i \leq k$, placing the vertices in $I$ from left to right in non-decreasing order by $\delta(v)$ gives an optimal ordering within this gap. Indeed, if there exists a pair of vertices $u, v \in I$ which are adjacent according to $\pi$ in gap $i$ with $\delta(v)>\delta(u)$ and $\pi(v)<\pi(u)$, we can swap $v$ and $u$ and obtain a linear arrangement of smaller cost. It follows that all of the vertices $v$ with equal force in gap $i$ will be placed consecutively. We can thus place all of the vertices of type $t$ as a contiguous block in gap $i$ without harming the optimality of the arrangement, since all of these vertices have the same force in gap $i$.

Lemma 2. The cost of any linear arrangement for $G$ is at least $\frac{m^{2}}{4 k^{2}}$.

Proof. It is not difficult to see that a graph $G$ with $v c(G)=k$ and $m$ edges attaining the minimum possible cost of a linear arrangement is the disjoint union of $k$ stars. Consider such a graph $G^{*}$, and let $\ell_{r}$ denote the number of edges in the $r$-th star of $G^{*}, 1 \leq r \leq k$. Then the cost of a minimum linear arrangement of $G^{*}$ is lower-bounded by

$$
M L A\left(G^{*}\right) \geq \sum_{r=1}^{k} \sum_{d=1}^{\left\lfloor\ell_{r} / 2\right\rfloor} 2 d \geq \sum_{r=1}^{k} \frac{\ell_{r}^{2}}{4} .
$$

The function $\sum_{r=1}^{k} \frac{\ell_{r}^{2}}{4}$ above is minimized when $\ell_{r}=\frac{m}{k}$ for all $1 \leq r \leq k$. Thus, $\operatorname{MLA}\left(G^{*}\right) \geq \frac{m^{2}}{4 k^{2}}$, and the lemma follows. 
Lemma 3. Let $M L A(G)$ denote the cost of the optimal arrangement for $G$, and let $M L A\left(G^{\prime}\right)$ denote the cost of the layout returned by Algorithm 1. Then $M L A\left(G^{\prime}\right) \leq M L A(G)+\frac{\varepsilon m^{2}}{4 k^{2}}$.

Proof. We note that Algorithm 1 considers only assignments of integral numbers of mega-vertices in each gap. However, it may be the case that any optimal ordering for $G$ contains fractional assignments of mega-vertices in some of the gaps. Consider such an optimal ordering $\pi_{o}$ for $G$. Let $\pi_{o}^{I}$ be an integral assignment in which the number of mega-vertices of each type in any gap of $\pi_{o}$ is rounded up/down to the next integral value. Thus, the total increase in the number of vertices in any gap is at most $s \cdot 2^{k}=\frac{\varepsilon m}{4 k^{2}(k+1)}$. Let lengtho $(e)$ be the cost incurred by $e \in E$ (or, the length of $e$ ) in $\pi_{o}$. Consider an iteration of Algorithm 1, in which it considers an integral assignment that corresponds to $\pi_{o}$. Then, length $h_{o}(e)$ is stretched by the additional vertices in $\pi_{o}^{I}$, in each gap that $e$ crosses, i.e., at most by $s \cdot 2^{k} \cdot(k+1)=\frac{\varepsilon m}{4 k^{2}}$. Algorithm 1 outputs an integral assignment of minimum cost, and the lemma follows.

Proof of Theorem 1: Observe that the running time of Algorithm 1 can roughly be bounded by the number of linear arrangements of $G^{\prime}$. Note that $\left|E^{\prime}\right|=\lceil m / s\rceil=O\left(2^{k} k^{3}\right)$ and $\left|V^{\prime}\right| \leq 2\left|E^{\prime}\right|$, as $G^{\prime}$ has no isolated vertices. Thus, the total running time of the algorithm can be bounded by $O^{*}\left(\left(2^{k} k^{3}\right) !\right)$. Furthermore, by Lemmas 2 and 3, we have

$$
\frac{M L A\left(G^{\prime}\right)}{M L A(G)} \leq 1+\frac{\varepsilon m^{2}}{4 k^{2} \cdot M L A(G)} \leq 1+\varepsilon,
$$

where $M L A\left(G^{\prime}\right)$ denotes the minimum cost of the layout returned by the algorithm, and $M L A(G)$ denotes the optimal cost. Thus, Algorithm 1 returns a $(1+\varepsilon)$-approximate solution in FPT time with respect to $(\varepsilon, k)$.

\section{MLA parameterized by Max-Leaf Number}

In this section we give an FPT algorithm for MLA parameterized by $k=$ $m \ell(G)$, for $k>1$. We start with some definitions. Given a graph $H=$ $\left(V^{\prime}, E^{\prime}\right)$, a subdivision of an edge $e^{\prime}=(u, v) \in E^{\prime}$ replaces $e^{\prime}$ by $E_{\text {sub }}\left(e^{\prime}\right)=$ $\left\{\left(u, v_{1}\right)\left(v_{1}, v_{2}\right) \ldots\left(v_{n_{e^{\prime}}}, v\right)\right\}$, for some $n_{e^{\prime}} \geq 1$. Thus, the edge $e^{\prime}$ becomes an edge-path, and we add to $H^{\prime} n_{e^{\prime}}$ vertices. We say that a graph $G$ is a subdivision of a graph $H$ if $G$ was generated by subdivision operations on the edges of $H$.

As shown in [16], if $m \ell(G)=k$ then $G$ is a subdivision of a graph $H$ on at most $4 k-2$ vertices. We call $H=\left(V^{\prime}, E^{\prime}\right)$, where $V^{\prime} \subseteq V$ and $\left|V^{\prime}\right|=k^{\prime} \leq 4 k-2$, the seed graph of $G$. Let $S \subseteq E^{\prime}$ denote the set of subdivided edges in $H$. Then, $V=V^{\prime} \cup\left(\cup_{e^{\prime} \in S}\left\{v_{1}, \ldots, v_{n_{e^{\prime}}}\right\}\right)$, and $E=E^{\prime} \backslash S \cup\left(\bigcup_{e^{\prime} \in S} E_{s u b}\left(e^{\prime}\right)\right)$. We say that a vertex $w \in V \backslash V^{\prime}$ belongs to $e^{\prime}$, where $e^{\prime}=(u, v) \in E^{\prime}$, if $w$ is on the path $\left(u, v_{1}, \ldots v_{n_{e^{\prime}}}, v\right)$ in $G$.

Our algorithm for MLA, parameterized by $m \ell(G)$, branches on an exhaustive set of solution patterns which has size bounded by a function of $k$. This set of 
patterns contains at least one optimal solution for the graph $G$, if one exists. Then, to determine when a solution pattern can be realized, we solve a linear integer program which outputs placements for all vertices of $G$ in a permutation that is consistent with the given pattern. A detailed description is given in Algorithm 2.

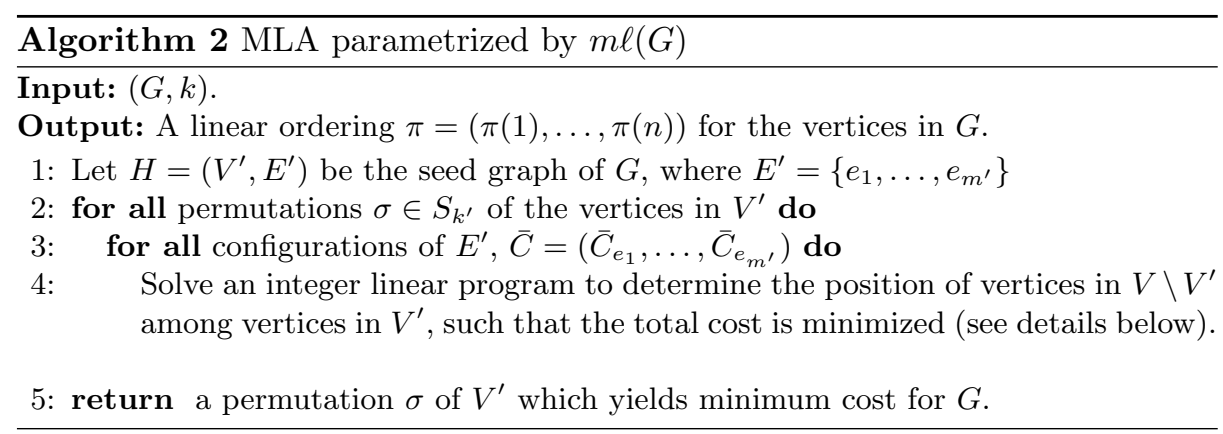

We define a permutation $\pi$ of the vertices in $G$, by extending a permutation $\sigma$ of the vertices in $H$. Given such a permutation $\sigma$, we now formulate an integer linear program to find the position of vertices in $V \backslash V^{\prime}$ among the vertices of $H$. Recall that any permutation $\sigma=\left(u_{1}, \ldots, u_{k^{\prime}}\right)$ defines $\left(k^{\prime}+1\right)$ gaps. Let $\pi$ be a permutation of $V$ that is consistent with $\sigma$. Let $\pi(v) \in[n]$ denote the position of vertex $v \in V$ in $\pi$, where $n=|V|$. We say that a vertex $w \in V \backslash V^{\prime}$ is placed in gap $i$ in $\pi, 1 \leq i \leq k^{\prime}-1$, if $\pi\left(u_{i}\right)<\pi(w)<\pi\left(u_{i+1}\right)$, where $u_{i}, u_{i+1} \in V^{\prime} ; w$ is placed in gap $0\left(k^{\prime}\right)$ if $\pi(w)<\pi\left(u_{1}\right)\left(\pi(w)>\pi\left(u_{k^{\prime}}\right)\right)$.

A configuration for an edge-path of $e^{\prime} \in E^{\prime}$ is a $\left(k^{\prime}+1\right)$-vector, $\bar{C}_{e^{\prime}}=$ $\left(c_{e^{\prime}, 0}, \ldots, c_{e^{\prime}, k^{\prime}}\right)$, in which the $i$-th entry is equal to ' 1 ' if gap $i$ contains a vertex in $e^{\prime}$, and ' 0 ' otherwise. Let $\left|E^{\prime}\right|=m^{\prime}$. Then, a configuration for $E^{\prime}$ is a set of $m^{\prime}$ configuration vectors for all edge-paths in $E^{\prime}$. We use in the integer program the variables $x_{e^{\prime}, i}$ to indicate the number of vertices on the edge-path of $e^{\prime} \in E^{\prime}$ in the $i$-th gap, $0 \leq i \leq k^{\prime}$. Let $\sigma \in S_{k^{\prime}}$ be a permutation of the vertices in $V^{\prime}$. We denote by $\operatorname{Cost}(G \mid \sigma, \bar{x})$ the total cost of the linear arrangement of the vertices in $G$, with the given permutation $\sigma$ and the variable values. This cost can be computed in FPT time, using Lemma 5 (see Section 3.1). Then our program can be formulated as follows.

\subsection{Analysis}

We now prove that Algorithm 2 yields an optimal solution. Our analysis crucially relies on the next two lemmas.

Lemma 4. Given a permutation $\sigma \in S_{k^{\prime}}$ for $V^{\prime}$, and the number of vertices of edge-path $e^{\prime}$ in gap $i, x_{e^{\prime}, i}, 0 \leq i \leq k^{\prime}$, there exists an optimal order for $V$ where each gap $i$ for which $x_{e^{\prime}, i}>0$ contains exactly one or two contiguous segments of $e^{\prime}$. 


\begin{tabular}{|c|c|c|c|}
\hline \multirow[t]{4}{*}{$\left(L P_{m \ell}\right):$} & minimize & $\operatorname{Cost}(G \mid \sigma, \bar{x})$ & \\
\hline & subject to: & $\sum_{i=0}^{k^{\prime}} x_{e^{\prime}, i}=n_{e^{\prime}}$ & $\forall e^{\prime} \in E^{\prime}$ \\
\hline & & $x_{e^{\prime}, i}=0$ & if $c_{e^{\prime}, i}=0$ \\
\hline & & $x_{e^{\prime}, i} \in\left\{1, \ldots, n_{e^{\prime}}\right\}$ & if $c_{e^{\prime}, i}=1$ \\
\hline
\end{tabular}

Lemma 5 (Homogeneity for $m \ell(G)$ ). There exists an optimal layout in which vertices in $V \backslash V^{\prime}$ that belong to the same edge-path appear consecutively in gap $i$, for all $0 \leq i \leq k^{\prime}$.

Proof. Given a permutation $\pi$ of the vertices in $G$, we show below that if the ordering of vertices in gap $i$ does not satisfy the contiguity property in the lemma then we can modify the order of vertices to satisfy the property, without increasing the total cost. Given the permutation $\pi$, we say that a vertex $v \in V \backslash V^{\prime}$ is a right-bend of an edge-path if its two neighbors, $u, w$, satisfy $\pi(u)<\pi(v)$ and $\pi(w)<\pi(v)$, i.e., both $u$ and $w$ appear to the left of $v$ in $\pi$. Similarly, $v$ is a left-bend if its two neighbors appear to its right in $\pi$.

In proving the lemma, we consider pairs of vertices in gap $i$ and show that we can swap the order of vertices in these pairs until we obtain a consecutive ordering of the vertices in each edge-path. Let $z, v \in V \backslash V^{\prime}$ be a pair of vertices that appear consecutively in $\pi$. Suppose that $z$ and $v$ belong to two different edge-paths, $e_{1}^{\prime}$ and $e_{2}^{\prime}$, respectively. Also, assume that the two neighbors of $z$ on $e_{1}^{\prime}$ are $r, x \in V$, and the two neighbors of $v$ on $e_{2}^{\prime}$ are $u, w \in V$. We distinguish between four cases.

(i) Neither $z$ nor $v$ is a bend. Let $\pi^{\prime}$ be the permutation resulting from a swap of $z$ and $v$ in $\pi=\left(v_{i_{1}}, \ldots, x, \ldots, u, \ldots v, z, \ldots, w, \ldots r, \ldots, v_{i_{n}}\right)$. Then, due to the swap, the distance between $z$ and $r(x)$ increases (decreases) by one; similarly, the distance between $v$ and $u(w)$ increases (decreases) by one. Thus, the overall change in the permutation cost is equal to zero.

(ii) Exactly one of $z, v$ is a bend. W.l.o.g, assume that $v$ is a right bend. (the proof is similar for the case where $v$ is a left bend). Suppose that $\pi(w)<$ $\pi(u)<\pi(v)$, and assume that the vertex $z$ precedes $v$ in gap $i$, i.e., we have $\pi=\left(v_{i_{1}}, \ldots, w \ldots, u, \ldots, z, v, \ldots, v_{i_{n}}\right)$. We show that swapping $z$ and $v$ does not increase the total cost. Let $\pi^{\prime}$ be the resulting permutation. Since $z$ is not a bend, it has two neighbors $x, r$, such that $\pi(x)<\pi(z)<\pi(r)$. Thus, after swapping $z$ and $v, z$ gets closer (by one) to $r$, while its distance from $x$ increases by one. On the other hand, due o the swap, $v$ gets closer to both $u$ and $w$. Hence, overall, we have that $\operatorname{cost}\left(\pi^{\prime}\right)-\operatorname{cost}(\pi)<0$. 
(iii) Suppose that $z, v$ are bends of the same direction. W.l.o.g., assume that both are right bends, and we have that $\pi(x)<\pi(r)<\pi(z), \pi(w)<\pi(u)<\pi(v)$. More precisely, let $\pi=\left(v_{i_{1}}, \ldots, x \ldots, w, \ldots, r, \ldots, u, \ldots z, v, \ldots, v_{i_{n}}\right)$. Then, it is easy to verify that $\operatorname{cost}\left(\pi^{\prime}\right)-\operatorname{cost}(\pi)=0$.

(iv) Suppose that $z, v$ are bends having opposite directions. W.l.o.g., assume that $v$ is a right bend and $z$ is a left bend. We further assume that $\pi(z)<\pi(r)<\pi(x)$, and $\pi(w)<\pi(u)<\pi(v)$, i.e., $\pi=$ $\left(v_{i_{1}}, \ldots, w \ldots, u, \ldots, z, v, \ldots r, \ldots, x, \ldots, v_{i_{n}}\right)$. Indeed, as a result of the swap, both $v$ and $z$ become closer to their neighbors. Hence, $\operatorname{cost}\left(\pi^{\prime}\right)-$ $\operatorname{cost}(\pi)<0$.

We can perform a sequence of the above swaps for pairs of vertices that belong to different edge-paths, until we have that all of the vertices on the same edge-path appear consecutively in gap $i$, without increasing the cost.

Lemma 6. Given a permutation $\sigma$ and the vector $\bar{x}, \operatorname{Cost}(G \mid \sigma, \bar{x})$ is linear and can be computed in FPT time.

Proof. We can write $\operatorname{Cost}(G \mid \sigma, \bar{x})=\sum_{i=0}^{k^{\prime}} \operatorname{Cost}($ gap $i \mid \sigma, \bar{x})$, where $\operatorname{Cost}($ gap $i \mid \sigma, \bar{x})$ is the contribution of gap $i$ to the total cost. Specifically, suppose that the two ends of gap $i$ are $x, y \in V^{\prime}$, where $\pi(x)<\pi(y)$. Let $\left(v_{1}, \ldots, v_{h}\right)$ be a segment of edge-path $e^{\prime}$ in gap $i$. W.l.o.g., we assume that $v_{1}$ is closer to $x$. Then we compute the contribution of this segment to the cost of gap $i$ as follows. We compute the cost incurred by each internal edge on this segment and add to that a term that depends on the type of the segment. By Lemma 4, we may assume that the segment $\left(v_{1}, \ldots, v_{h}\right)$ is contiguous.

(a) If the segment is straight (i.e., has no bend in gap $i$ ), we add the distance from $v_{1}$ to $x$ and from $v_{h}$ to $y$. This term would then partially account for the cost incurred by the edges connecting the two ends of the segment, $v_{1}$ and $v_{h}$, to their neighbors in other gaps.

(b) If the segment has a right bend, we add the distance between $v_{1}$ and $x$ plus the distance between $v_{h}$ and $x$.

(c) If the segment has a left bend, we add the distance between $v_{1}$ and $y$ and the distance between $v_{h}$ and $y$.

Thus, it suffices to show that $\operatorname{Cost}($ gap $i \mid \sigma, \bar{x})$ is linear and can be computed in FPT time. We now show how to order optimally the segments in gap $i$. Let $B_{\ell}, B_{r}$ and $S$ denote the sets of segments of types left-bend, right-bend and straight in gap $i$, respectively. We denote by $z_{i}=\sum_{e^{\prime} \in E^{\prime}} x_{e^{\prime}, i}$ the number of vertices assigned to gap $i$. We give the proofs of the next claims in the Appendix.

Claim 2 The cost incurred by any segment of type $S$ in gap $i$ is equal to $z_{i}$.

Claim 3 Given a set of segments in $B_{r}$ in gap $i$, of lengths $x_{e_{1}, i} \leq x_{e_{2}, i} \leq \cdots \leq$ $x_{e_{r}, i}$, the minimum total cost incurred by these segments in gap $i$ is given by $r \cdot x_{e_{1}, i}+(r-1) x_{e_{2}, i}+\cdots+x_{e_{r}, i}$. Similarly, given a set of segments in $B_{\ell}$, of lengths $x_{e_{1}, i} \leq x_{e_{2}, i} \leq \cdots \leq x_{e_{\ell}, i}$, the minimum cost incurred by these segments is $\ell \cdot x_{e_{1}, i}+(\ell-1) x_{e_{2}, i}+\cdots+x_{e_{\ell}, i}$. 
Thus, letting $\left|B_{r}\right|=r$ and $\left|B_{\ell}\right|=\ell$, by Claims 2 and 3 we have that the total cost of gap $i$ is given by

$$
\operatorname{Cost}(\operatorname{gap} i \mid \sigma, \bar{x})=|S| \cdot \sum_{e^{\prime} \in E^{\prime}} x_{e^{\prime}, i}+\sum_{j=1}^{r}(r-j+1) x_{e_{j}, i}+\sum_{j=1}^{\ell}(\ell-j+1) x_{e_{j}, i},
$$

where $e_{1}, \ldots, e_{r}$ are the edge-paths having in gap $i$ segments in $B_{r}$, and $e_{1}, \ldots, e_{\ell}$ are the edge-paths having in gap $i$ segments in $B_{\ell}$. For a vector $\bar{x}$ that is consistent with a given configuration of $E^{\prime}$, we have that the values of $r$ and $\ell$ in (1) are fixed, thus $\operatorname{Cost}($ gap $i \mid \sigma, \bar{x})$ is linear, and so is $\operatorname{Cost}(G \mid \sigma, \bar{x})$. Hence, we can solve the integer program in FPT time (see, e.g., $[17,15]$ ).

We summarize in the next result.

Theorem 4. There is an FPT algorithm for MLA parameterized by the maximum leaf number.

Proof. Clearly, by Lemmas 4 and 5, our algorithm exhaustively searches over the set of solution patterns which contains an optimal one. It remains to show that the algorithm runs in FPT time. We note that the two outer loops of Algorithm 2 require $O\left(k^{\prime} ! \cdot 2^{k^{3}}\right)$ iterations, each requires solving an integer linear program having $O\left(\left|E^{\prime}\right| \cdot k^{\prime}\right)=O\left(k^{3}\right)$ variables. This gives the statement of the theorem.

\section{MLA parameterized by Edge-Clique-Cover Number}

In this section we show that MLA parameterized by the edge clique cover number of the input graph is in FPT. More precisely, we prove the following.

Theorem 5. There is an $O^{*}\left(2^{k} !\right)$-time algorithm for $M L A$ where $k=e e c(G)$ is the edge clique cover number of the input graph $G$.

We define the type of node $u \in V$ to be $N[u]=N(u) \cup\{u\}$. Note that our notion of type here is different from the one we use in Section 2, as vertices of the same type are necessarily adjacent. Nevertheless, the two different definitions are conceptually very similar, as we can prove a certain homogeneity lemma for this notion of type as well.

Lemma 7 (Homogeneity for ecc $(G)$ ). There exists an optimal vertex ordering which is homogenous. That is, an ordering in which vertices of each type appear consecutively.

Proof of Theorem 5 [assuming Lemma 7]: By Lemma 7, there exists an optimal solution in which vertices of each type appear consecutively. Observe that in any such homogenous ordering, the ordering of vertices of the same type can be arbitrary. That is, reordering vertices of a given type does not affect the total edge lengths of the ordering. Now, it is well known that a graph with edge clique cover number at most $k$ has at most $2^{k}$ different types [13]. Thus, our 


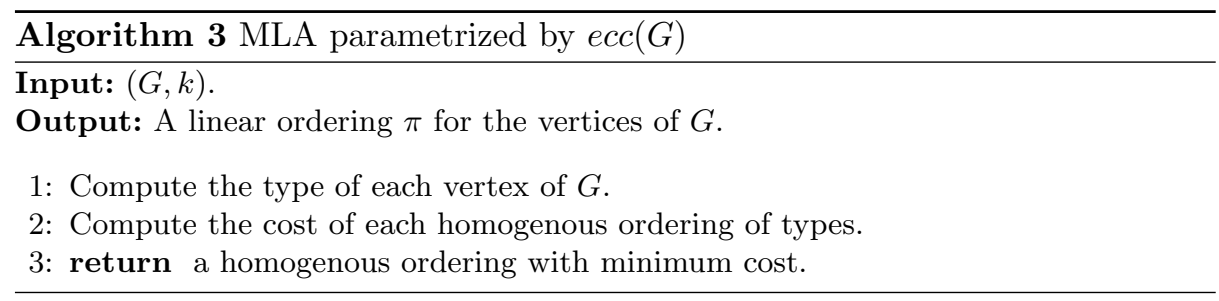

algorithm searches through all $O\left(2^{k}\right.$ !) homogenous vertex orderings and outputs the best one.

To prove Lemma 7, we introduce some additional notation. For an ordering $\pi=V \rightarrow\{1, \ldots, n\}$ and a pair of vertices $u, v \in V$ such that $\pi(u)<\pi(v)$, we let $\overleftarrow{\pi_{u, v}}$ and $\overrightarrow{\pi_{u, v}}$ denote the following permutations:

$$
\overleftarrow{\pi_{u, v}}(x)= \begin{cases}\pi(x) & \text { if } \pi(x) \leq \pi(u) \text { or } \pi(v)<\pi(x) \\ \pi(u)+1 & \text { if } x=v \\ \pi(x)+1 & \text { if } \pi(u)<\pi(x)<\pi(v)\end{cases}
$$

and

$$
\overrightarrow{\pi_{u, v}}(x)= \begin{cases}\pi(x) & \text { if } \pi(x)<\pi(u) \text { or } \pi(v) \leq \pi(x), \\ \pi(v)-1 & \text { if } x=u \\ \pi(x)-1 & \text { if } \pi(u)<\pi(x)<\pi(v) .\end{cases}
$$

Thus, $\overleftarrow{\pi_{u, v}}$ is the vertex ordering obtained from $\pi$ by placing $v$ directly after $u$, and $\overrightarrow{\pi_{u, v}}$ is the ordering obtained from $\pi$ by placing $u$ directly before $v$.

Lemma 8. Let $\pi=V \rightarrow\{1, \ldots, n\}$ be an optimal vertex ordering, and let $u$ and $v$ be two vertices of the same type with $\pi(u)<\pi(v)$. Then both $\overleftarrow{\pi_{u, v}}$ and $\overrightarrow{\pi_{u, v}}$ are optimal as well.

Proof. Define three set of vertices $A=\{x \in V: \pi(x)<\pi(u)\}, B=\{x \in$ $V: \pi(u)<x<\pi(v)\}$, and $C=\{x \in V: \pi(v)<\pi(x)\}$, and consider the two quantities $\overleftarrow{\Delta}=\operatorname{cost}\left(\overleftarrow{\pi_{u, v}}\right)-\operatorname{cost}(\pi)$ and $\vec{\Delta}=\operatorname{cost}\left(\overrightarrow{\pi_{u, v}}\right)-\operatorname{cost}(\pi)$. As $\pi$ is optimal, both these quantities are non-negative, i.e. $\overleftarrow{\Delta} \geq 0$ and $\vec{\Delta} \geq 0$. If the lemma were false, at least one of these quantities would be strictly positive, i.e. we would either have $\overleftarrow{\Delta}>0$ or $\vec{\Delta}>0$. Aiming towards a contradiction let us assume that $\overleftarrow{\Delta}>0$ (the proof in case $\vec{\Delta}>0$ is symmetric)

Let us examine which edges contribute to $\overleftarrow{\Delta}$, i.e. which edges $\{x, y\} \in E$ have different lengths in $\overrightarrow{\pi_{u, v}}$ and $\pi\left(\left|\overrightarrow{\pi_{u, v}}(x)-\overrightarrow{\pi_{u, v}}(y)\right| \neq|\pi(x)-\pi(y)|\right)$. Clearly, edges in $E(A, A), E(B, B), E(C, C)$ and $E(A, C)$ do note contribute to $\overleftarrow{\Delta}$. All other edge lengths are different in the two orderings. The length of each edge in $E(v, C)$ increases by $|B|$ in $\overleftarrow{\pi_{u, v}}$ when compared to its length in $\pi$, while the length of each edge in $E(v, A)$ decreases by $|B|$. Similarly, the length of each edge in $E(A, B)$ increases by 1 , while the length of each edge in $E(B, C)$ decreases by 1 . 
What remains to account for are the edges involving $u$ and $v$. Let $\ell(u, B)=$ $\sum_{b \in B}|\pi(u)-\pi(b)|$ and $\ell(v, B)=\sum_{b \in B}|\pi(v)-\pi(b)|$ denote the total length of the edges of $E(u, B)$ and $E(v, B)$ with respect to $\pi$. Observe that as $u$ and $v$ are twins (and are thus adjacent to the same set of vertices in $B$ ), the total length of all edges of $E(v, B)$ becomes $\ell(u, B)$ in $\overleftarrow{\pi_{u, v}}$, while the length of each edge of $E(u, B)$ increases by 1 . Thus, the total contribution of edges in $E(u, B) \cup E(v, B)$ to $\overleftarrow{\Delta}$ is $|E(u, B)|+\ell(u, B)-\ell(v, B)$. Finally, the last edge contributing to $\overleftarrow{\Delta}$ is the edge $\{u, v\}$ itself (which necessarily exists as $u$ and $v$ are from the same type) whose length decreases by $|B|$ in $\overleftarrow{\pi}_{u, v}$ when compared to $\pi$.

Summing up all these contributions, we get the following equality for $\overleftarrow{\Delta}$ :

$$
\begin{aligned}
\overleftarrow{\Delta} & =|E(v, C)| \cdot|B|+|E(A, B)|+\ell(u, B)+|E(u, B)| \\
& -|E(v, A)| \cdot|B|-|E(B, C)|-\ell(v, B)-|B| .
\end{aligned}
$$

Symmetrically, a similar calculation will give us the following equation for $\vec{\Delta}$ :

$$
\begin{aligned}
\vec{\Delta} & =|E(u, A)| \cdot|B|+|E(B, C)|+\ell(v, B)+|E(v, B)| \\
& -|E(u, C)| \cdot|B|-|E(A, B)|-\ell(u, B)-|B| .
\end{aligned}
$$

Now as $u$ and $v$ are twins, we know that $|E(u, A)|=|E(v, A)|$ and $|E(u, C)|=$ $|E(v, C)|$. Furthermore, we also have $|B| \geq|E(u, B)|=\mid E(v, B)$. Thus, using equations (2) and (3), and our assumption that $\overleftarrow{\Delta}>0$ and $\vec{\Delta} \geq 0$, we get

$$
\begin{array}{r}
|E(v, C)| \cdot|B|+|E(A, B)|+\ell(u, B)+|E(u, B)|> \\
|E(v, A)| \cdot|B|+|E(B, C)|+\ell(v, B)+|B| \geq \\
|E(u, A)| \cdot|B|+|E(B, C)|+\ell(v, B)+|E(v, B)| \geq \\
|E(u, C)| \cdot|B|+|E(A, B)|+\ell(u, B)+|B| \geq \\
|E(v, C)| \cdot|B|+|E(A, B)|+\ell(u, B)+|E(u, B)|
\end{array}
$$

our desired contradiction.

Proof of Lemma 7: Let $\pi$ be an optimal vertex ordering. If $\pi$ is not homogenous we can use Lemma 8 repeatedly to transform it into one. The lemma thus follows.

\section{Summary and Open Problems}

We have shed light on the complexity of MLA for structural parameterizations stronger than treewidth, including a nice example of a successful combination of parameterization and approximation. We believe our algorithmic strategy in this may be applicable elsewhere, but can only so far show its applicability for the three parameters above. Can our results be extended to the aggregate parameterization based on treewidth? Furthermore, the aim of this paper was only at qualitative FPT results. We leave the best running times for such FPT algorithms as an open problem. 


\section{References}

1. C. Ambühl, M. Mastrolilli, and O. Svensson. Inapproximability results for maximum edge biclique, minimum linear arrangement, and sparsest cut. SIAM J. Comput., 40(2):567-596, 2011.

2. H. L. Bodlaender, M. R. Fellows, and M. T. Hallett. Beyond NP-completeness for problems of bounded width: hardness for the $\mathrm{W}$ hierarchy. In Proceedings of the 26th Annual Symposium on the Theory of Computing (STOC), pages 449-458, 1994.

3. H. L. Bodlaender, F. V. Fomin, A. M. C. A. Koster, D. Kratsch, and D. M. Thilikos. A note on exact algorithms for vertex ordering problems on graphs. Theory of Computing Systems, 50(3):420-432, 2012.

4. M. Charikar, M. T. Hajiaghayi, H. J. Karloff, and S. Rao. $\ell_{2}^{2}$ spreading metrics for vertex ordering problems. In SODA, pages 1018-1027, 2006.

5. U. Feige and J. R. Lee. An improved approximation ratio for the minimum linear arrangement problem. Inf. Process. Lett., 101(1):26-29, 2007.

6. M. R. Fellows, F. V. Fomin, D. Lokshtanov, F. A. Rosamond, S. Saurabh, S. Szeider, and C. Thomassen. On the complexity of some colorful problems parameterized by treewidth. Information and Computation, 209(2):143-153, 2011.

7. M. R. Fellows, D. Hermelin, F. Rosamond, and H. Shachnai. Tractable parameterizations for the minimum linear arrangement problem. full version. http: //www.cs.technion.ac.il/ hadas/PUB/MLA_FHRS.pdf/.

8. M. R. Fellows, B. M. P. Jansen, and F. A. Rosamond. Towards fully multivariate algorithmics: Parameter ecology and the deconstruction of computational complexity. European Journal of Combinatorics, 34(3):541-566, 2013.

9. M. R. Fellows, D. Lokshtanov, N. Misra, M. Mnich, F. A. Rosamond, and S. Saurabh. The complexity ecology of parameters: An illustration using bounded max leaf number. Theory Comput. Syst., 45(4):822-848, 2009.

10. M. R. Fellows, D. Lokshtanov, N. Misra, F. A. Rosamond, and S. Saurabh. Graph layout problems parameterized by vertex cover. In ISAAC, pages 294-305, 2008.

11. H. Fernau. Parameterized algorithmics for linear arrangement problems. Discrete Applied Mathematics, 156(17):3166-3177, 2008.

12. M. R. Garey and D. S. Johnson. Computers and intractability: a guide to the theory of NP-completeness. W. H. Freeman, 1979.

13. J. Gramm, J. Guo, F. Hüffner, and R. Niedermeier. Data reduction and exact algorithms for clique cover. ACM Journal of Experimental Algorithmics, 13, 2008.

14. G. Gutin, A. Rafiey, S. Szeider, and A. Yeo. The linear arrangement problem parameterized above guaranteed value. Theory Comput. Syst., 41(3):521-538, 2007.

15. R. Kannan. Minkowski's convex body theorem and integer programming. Mathematics of Operations Research, 12:415-440, 1987.

16. D. J. Kleitman and D. B. West. Spanning trees with many leaves. SIAM J. Discrete Math., 4(1):99-106, 1991.

17. H. Lenstra. Integer programming with a fixed number of variables. Mathematics of Operations Research, 8:538-548, 1983.

18. S. Rao and A. W. Richa. New approximation techniques for some linear ordering problems. SIAM J. Comput., 34(2):388-404, 2004. 


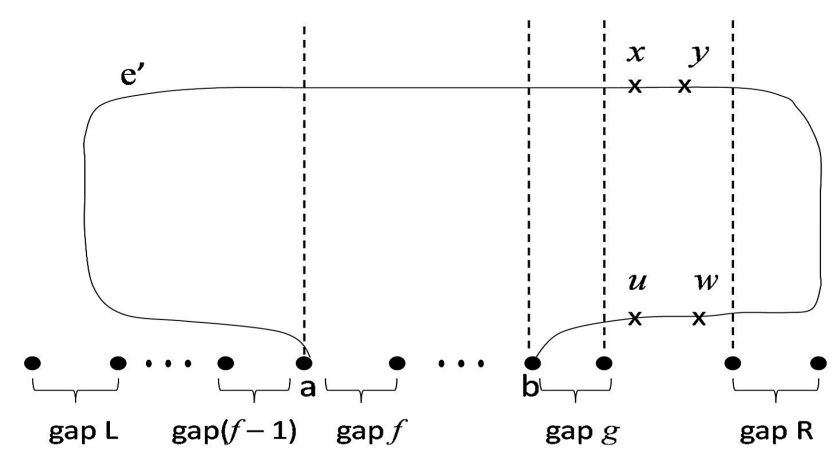

Fig. 1. The layout of an edge-path connecting $a$ and $b$ : Case 1. in the proof of Lemma 4

\section{A Some Proofs}

Proof of Lemma 4: Suppose that the edge-path of $e^{\prime}$ contains the vertices $\left(a, v_{1}, \ldots, v_{h}, b\right)$, where $a, b \in V^{\prime}$ and $v_{1}, \ldots, v_{h} \in V \backslash V^{\prime}$. Thus, $e^{\prime}$ incurs cost for $h+1$ edges. For any edge on this path, the cost of connecting two neighboring vertices $v_{i}, v_{j}$ that appear in different gaps is 2 or higher, since at least one vertex in $V^{\prime}$ separates between these vertices in the vertex ordering, $\pi$. We show below that there is an optimal ordering for $V$ which assigns in each gap either a single contiguous segment, or two contiguous segments of $e^{\prime}$. Let $\sigma(a)=f$ and $\sigma(b)=g$, and assume w.l.o.g. that $1 \leq f<g \leq k^{\prime}$. Let $L=\min \left\{i \mid x_{e^{\prime}, i}>0\right\}$, and $R=\max \left\{i \mid x_{e^{\prime}, i}>0\right\}$, i.e., $L$ and $R$ are the indices of the leftmost and rightmost gaps that contain vertices of $e^{\prime}$, respectively. We first consider the cost incurred by internal edges, i.e., edges connecting neighboring vertices of $e^{\prime}$ that are placed in the same gap.

W.l.o.g., we may assume that $x_{e^{\prime}, i}>1$. We distinguish between the following cases.

1. $R \geq g$ and $L \leq f-1$ (see Figure 1). Then we consider four types of gaps.

(i) In any gap $f \leq i \leq g-1, e^{\prime}$ must contain a vertex $z \in\left\{v_{1}, \ldots, v_{h}\right\}$ having neighbor in gap $j \geq i+1$, and a vertex $x \in\left\{v_{1}, \ldots, v_{h}\right\}$ having neighbor in gap $j^{\prime} \leq i-1$, by the vector $\bar{x}_{e^{\prime} i}$. We can take a contiguous segment of length $t=x_{e^{\prime}, i}$, given by $\left(v_{s}, \ldots, v_{s+t-1}\right)$, and assign these vertices consecutively from left to right, i.e., $\pi\left(v_{i}\right)=\pi\left(v_{i-1}\right)+1$, for all $s+1 \leq i \leq s+t-1$. Thus, the cost incurred by each internal edge is 1 . 


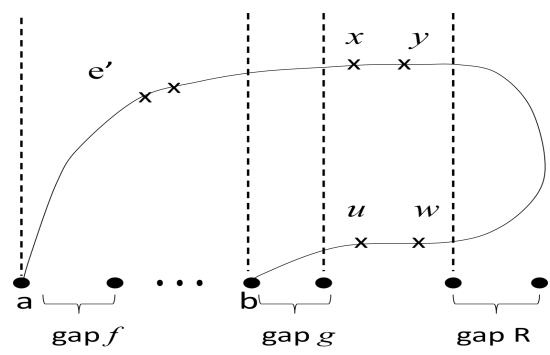

Fig. 2. Case 2. in the proof of Lemma 4

(ii) In gap $R, e^{\prime}$ must contain at least two vertices which have neighbors in gaps to the left of $R$. As argued above, if an edge between two vertices in $e^{\prime}$ is external (i.e., its ends are placed in different gaps), the cost incurred by this edge is at least 2 . Let $x_{e^{\prime}, R}=q$, and consider a contiguous segment $\left(x_{p}, \ldots, x_{p+q-1}\right)$. Let $d=\lfloor q / 2\rfloor$. We choose $v_{p+d}$ as a right-bend and order the vertices of the segment from left to right as follows: $\left(v_{p}, v_{p+q-1}, v_{p+1}, v_{p+q-2}, \ldots, v_{p+d-1}, v_{p+d+1}, v_{p+d}\right)$. Then, the cost incurred by each internal edge in this segment is at most 2 .

(iii) Similarly, we can order the vertices of a contiguous segment in gap $L$ with a single left-bend, such that each internal edge incurs at most the cost 2 .

(iv) If $R>g$, or $L<f-1$, then there exist some gap $i$ in which two vertices of $e^{\prime}$ must have neighbors in gaps to the right of $i$, and two vertices must have neighbors to the left of $i$ (see the vertices $x, u$ and $y, w$ in Figure 1). In each such gap, we can assign two contiguous segments of $e^{\prime}$ and order each as a straight line. The cost incurred by each internal edge in these segments is 1 .

2. $R \geq g$ and $L>f-1$. Similar to Case 1., except that there is no left-bend (see Figure 2).

3. $R<g$ and $L \leq f-1$. Similar to Case 1., except that there is no rightbend.

4. $R<g$ and $L>f-1$. In this case, the edge-path of $e^{\prime}$ has neither a right-bend nor a left-bend. Therefore, we are in sub-case $(i)$ of 1 .

Hence, we may assume that any gap $i$ for which $x_{e^{\prime}, i}>0$ contains either one or two contiguous sgements of the edge-path of $e^{\prime}$. It remains to select the contiguous segments placed in these gaps. We now consider the cost incurred by external edges. We note that the total cost incurred by these edges is minimized by assigning the contiguous segments of the edge-path such that the left (right) neighbor of each segment is as closest as possible from the left (right). Indeed, this follows from the fact that the cost of each external adge between neighbors on an edge-path depends on the total number of vertices in the gaps separating between these neighbors. We can obtain such optimal assignment by placing 
the segments continuously, starting from $a$, proceeding towards $L$, then towards $R$ and finally to $b$.

Proof of Claim 2: Consider an order in which the vertices of the segment appear consecutively in gap $i$. Let $\left(v_{1}, \ldots, v_{h}\right)$ be a segment in $S$ and assume, w.l.o.g, that $v_{1}$ is closer to $x$, the left end of gap $i$. By Lemma 5 , we may assume that the vertices on this segement appear consecutively in gap $i$. Then the cost incurred by this segment is given by the length of this segment plus the distance between $v_{1}$ and $x$ plus the distance between $v_{h}$ and $y$, which sum to $z_{i}$.

Proof of Claim 3: We give the detailed proof for $B_{r}$. The proof is similar for $B_{\ell}$. By Lemma 5, we may assume that each segment in $B_{r}$ is assigned consecutively in gap $i$. Since each end of a segment in $B_{r}$ has a neighbor in some gap $j<i$, by an interchange argument, the minimum cost for these segments is achieved by assigning them from left to right in non-decreasing order of lengths, i.e., the leftmost segment in gap $i$ belongs to $e_{1}$ in $B_{r}$, the next one belongs to $e_{2}$ in $B_{r}$ and so on.

We assign the segments of $B_{\ell}$ in gap $i$ similarly, from right to left, by placing the shortest among them to be the rightmost in gap $i$, the second segment in $B_{\ell}$ to its left and so on. 